\title{
Anaesthesia and handling stress effects on pigmentation and monoamines in Arctic charr
}

\author{
Tobias Backström • Martina Heynen • Eva Brännäs • \\ Jan Nilsson • Svante Winberg • Carin Magnhagen
}

Received: 21 April 2016 / Accepted: 5 December 2016 / Published online: 4 January 2017

(C) The Author(s) 2017. This article is published with open access at Springerlink.com

\begin{abstract}
Stress responsiveness differs between individuals and is often categorized into different stress coping styles. Using these stress coping styles for selection in fish farming could be beneficial, since stress is one main factor affecting welfare. In Arctic charr (Salvelinus alpinus) carotenoid pigmentation is associated with stress responsiveness and stress coping styles. Thus this could be an important tool to use for selection of stress resilient charr. However, anaesthetics seem to affect carotenoid pigmentation, and it would be better if the method for selection could be implemented during normal maintenance, which usually includes anaesthetics. Therefore, this study investigated how the use of anaesthetics affected carotenoid pigmentation, i.e. number of spots, over time compared to no-anaesthetic treatment. Additionally, the stress indicators monoamines and glucocorticoids were investigated. The results indicate that the anaesthetic MS-222 affects
\end{abstract}

Electronic supplementary material The online version of this article (doi:10.1007/s10641-016-0563-0) contains supplementary material, which is available to authorized users.

T. Backström $(\bowtie) \cdot$ E. Brännäs · J. Nilsson $\cdot$ C. Magnhagen Department of Wildlife, Fish, and Environmental Studies, Swedish University of Agricultural Sciences, Umeå, Sweden e-mail: tobias.backstrom@slu.se

\section{Heynen}

Department of Ecology and Environmental Science, Umeå University, Umeå, Sweden

S. Winberg

Department of Neuroscience, Uppsala University, Uppsala, Sweden number of spots on the right side. This anaesthetic also increased dopaminergic activity in the telencephalon. Both brain dopaminergic and serotonergic activity was associated with spottiness. Further, behaviour during anaesthetization was associated with spots on the left side, but not the right side. Repetition of the same treatment seemed to affect spot numbers on the right side. In conclusion, this study shows that inducing stress in charr affects the carotenoid spots. Thus, it is possible to use anaesthetics when evaluating spottiness although careful planning is needed.

Keywords Anaesthesia - Carotenoid pigmentation . Lateralization $\cdot$ Monoamine $\cdot$ Salmonid $\cdot$ Stress

\section{Introduction}

Intraspecific divergence in stress response profiles is common among various animal taxa. This divergence in stress responses is often related to specific behavioural traits and has been referred to as stress coping styles. Typically, there are two extremes, namely proactive and reactive coping style (Koolhaas et al. 1999; Koolhaas et al. 2007). Proactive individuals are more aggressive, have a higher general activity, forming behavioural routines more easily, and respond to stress with a lower hypothalamic-pituitary-adrenal (HPA) axis reactivity as compared to reactive individuals (Koolhaas et al. 1999; Koolhaas et al. 2007). In recent years, a plethora of studies have shown that stress coping styles are evident in teleost fish (Øverli et al. 2005; Schjolden and 
Winberg 2007; Koolhaas et al. 2010; Conrad et al. 2011; Castanheira et al. 2015).

To reduce within-group variance and hence number of animals used would be beneficial, for example, in growth studies (Erhard et al. 1997). This could be achieved by selection of animals based on stress coping style. Further, if personality is a heritable trait, it could be beneficial to include this trait in the selection of farmed animals. Fish are relatively new in farming industry compared to terrestrial animals and plants, and intensive fish farming just started during the last century (Duarte et al. 2007). Welfare is an important issue within aquaculture and relates to stress coping styles (Huntingford et al. 2006). For instance, selection of fast-growing fish might lead to more aggressive and risk-taking fish (i.e. proactive copers), which could impair welfare by increased fighting (Huntingford and Adams 2005). Thus, selection using specific stress coping styles could be beneficial for aquaculture.

Stress coping styles are usually evaluated using tests such as confinement, dominance, and resident-intruder (Koolhaas et al. 1999). However, these tests are very time consuming and hence not practical if large numbers of individuals would need to be screened. Therefore there is a need for better and faster methods to test for stress coping styles. Interestingly, animal's visual appearance often signals condition or social status (Hill 2011). In vertebrates the two pigment groups, carotenoids and melanins, cause a colour variation that sometimes reflect physiological condition. More melanised animals seem to be more stress-resilient (Ducrest et al. 2008). This has been reported in teleost fish, such as Atlantic salmon (Salmo salar) and rainbow trout (Oncorhynchus mykiss), in which fish with more melaninbased skin spots have a lower physiological stress response than the fish with few spots (Kittilsen et al. 2009). However, social subordination induces skin darkening in salmonids (O'Connor et al. 1999; O'Connor et al. 2000; Eaton and Sloman 2011) including the Arctic charr (Salvelinus alpinus) (Höglund et al. 2000; Höglund et al. 2002). Thus, in Arctic charr darker animals do not seem to be more stress resilient. Instead, a recent study reported that the carotenoid pigmentation in Arctic charr could be used as an indicator of physiological stress responses (Backström et al. 2014). Additionally, socially subordinate charr have more carotenoid spots compared to dominant individuals (Backström et al. 2015a). Therefore, carotenoid pigmentation, rather than melanin-based pigmentation, seems to be signalling stress in Arctic charr. We predict that carotenoid-based pigmentation in Arctic charr could be usable as a tool for predicting stress responsiveness. Such an assessment could be made during other, routine evaluation of brood stock, such as weighing and measuring. These procedures, however, usually involve anaesthetics; however, anaesthetics may increase the number of carotenoid spots (Backström et al. 2015b). There is also an association between pigmentation and monoaminergic activity (Backström et al. 2015c). The monoaminergic activity of serotonin (5-hydroxytryptamine; 5-HT), dopamine (DA), and norepinephrine (NE) are all physiological stress indicators (Blanchard et al. 2001) and are also evident in teleost fish (Summers and Winberg 2006).

Based on these earlier studies, we investigated how carotenoid spots were affected by the photographing procedure on two consecutive days using or not using anaesthetics in all possible combinations (without anaesthetics during first, second, or both days). We monitored the behaviour during anaesthesia and the physiological stress responses of plasma cortisol and brain monoamines on the final day to get a better understanding of the specific stress responses. Specifically, two hypotheses were tested: (1) spots were affected by consecutive use of anaesthetic compared to no-anaesthetic, and (2) spots were correlated to stress responsiveness independent of treatment.

\section{Material and methods}

\section{Experimental animals and location}

This experiment was done at the Umeå Marine Research Station (UMF) using 1 year old juvenile Arctic charr from the $7^{\text {th }}$ generation of the Swedish Arctic charr breeding programme [Arctic superior, for details on the programme see Nilsson et al. (2010)]. The fish were transported to UMF several months before the experiment, and were kept in tanks supplied with running brackish water (3-4\%o) from the Bothnian Bay (temperature range 5 to $10{ }^{\circ} \mathrm{C}$, photoperiod $12 \mathrm{~h}$ light $/ 12 \mathrm{~h}$ dark). The fish were fed commercial pellets (BioMar; $4 \mathrm{~mm}$, www.biomar.com) at $1.0-1.5 \%$ of body mass (MB) per day depending on temperature. The experiment was done in May-June 2012 with a methodology approved by the Umeå Animal Research Ethical Committee. 
Experiment setup

On day 1, fish (body mass: $141.2 \pm 21.4 \mathrm{~g}$, fork length $\left(\mathrm{L}_{\mathrm{F}}\right): 23.9 \pm 1.1 \mathrm{~cm}$, mean $\pm \mathrm{SD}, N=32$ ) were netted from the stock, photographed on both sides as described by Backström et al. (2015a) using a Canon EOS 500D digital camera and a box with a transparent lid. Immediately after photographing, fish were anaesthetized with Tricaine methane sulfonate (MS-222, $0.15 \mathrm{~g} / \mathrm{L}$ ), weighed and measured. The fish were not anaesthetized prior to photographing based upon the apparent spot inducing effects by anaesthetics in Arctic charr (Backström et al. 2015b). Isolation of individual fish was created by separating experimental aquaria $(170 \mathrm{~L}$, $95 \times 41 \times 44 \mathrm{~cm}$ ) into four equally-sized $42.5 \mathrm{~L}$ compartments using removable dark PVC walls. The fish were allowed to acclimate for one week during which they were hand-fed commercial pellets. Each aquarium was continuously supplied with running water from the Bothnian Bay $\left(5-10^{\circ} \mathrm{C}\right)$, and the full spectrum daylight light was turned on at 06:00 and off at 18:00 h $(12 \mathrm{~h}$ light/12 h dark).

After the isolation, fish were divided into four treatment groups aimed at deducing how different handling procedures during photographing would affect carotenoid pigmentation and subsequent stress responses. Earlier results show that anaesthesia increases number of spots (Backström et al. 2015b), and therefore this procedure was aimed at deducing if this effect was sustained over time and/or exacerbated by repeated treatment. MS-222 was used as anaesthetic because it is one of two anaesthetics allowed in the European Union (EEC Council regulation 2377/90) and is the one used in the Swedish Arctic charr aquaculture. The four treatment groups were: 1) being photographed without anaesthesia ( $\mathrm{N}$ for non-anaesthetic) on day 8 and without anaesthesia (N) on day 9 (group $\mathrm{NN}, N=8$ ), 2) being photographed without anaesthesia on day 8 and with anaesthesia (A for anaesthetic) on day 9 (group NA, $N=8$ ), 3) being photographed with anaesthesia on day 8 and without anaesthesia on day 9 (group $\mathrm{AN}, N=8$ ), and 4) being photographed with anaesthesia on day 8 and with anaesthesia on day 9 (group AA, $N=8$ ).

During anaesthesia, the fish were transferred to a 17L bucket (Ø $36 \mathrm{~cm}$ ) filled with $4 \mathrm{~L}$ of a low dose of MS$222(0.15 \mathrm{~g} / \mathrm{L})$. The behaviour during the anaesthetisation process was recorded via a camcorder for further analysis [see below and Backström et al. (2015b)]. Fish were kept in the solution until they lost equilibrium and had no response to tactile stimuli. Immediately following this stage, the fish were photographed (see description above). The fish without anaesthesia were taken immediately from the aquaria to be photographed.

After photographing on day 9, the fish were sacrificed in a high dose of MS-222 $(0.30 \mathrm{~g} / \mathrm{L})$. Blood was immediately sampled via a heparinized syringe from the caudal vein, then the fish was decapitated and the brain dissected out. Sex was determined by visual inspection of gonads. The blood was subsequently centrifuged, the plasma collected and stored at $-20{ }^{\circ} \mathrm{C}$ until further analysis. The brains were divided into the easily identified telencephalon, cerebellum, optic tectum and brainstem (including hypothalamus) and were stored at $-80{ }^{\circ} \mathrm{C}$ until further analysis.

Image analysis

Photographs were analysed as described and validated earlier (see Fig. 1) (Backström et al. 2015a). Briefly, carotenoid-based spots were counted systematically in a rectangle $(2 \mathrm{~cm} \times 10 \mathrm{~cm})$ on each side of the fish using ImageJ (Rasband, W.S., ImageJ, U. S. National Institutes of Health, Bethesda, Maryland, USA, http://imagej.nih.gov/ij/, 1997-2012.).

\section{Assays}

Plasma was analysed for cortisol using a commercial enzyme linked immunosorbent assay (ELISA) kit (product \# 402710, Neogen Corporation, Lexington, USA).
Fig. 1 Typical photograph from the experiment. White arrows indicate typical spots that were counted

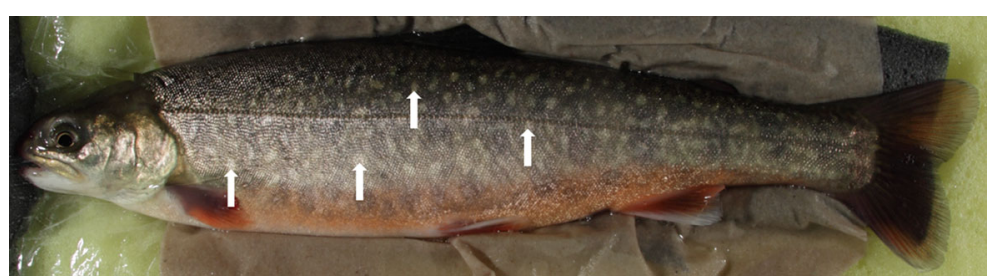


Each sample was run in duplicate during a single assay with an intra-assay coefficient of variation of $1.30 \%$.

Tissue levels of 5-HT and the 5-HT metabolite, 5hydroxyindoleacetic acid (5-HIAA), DA and the DA metabolite 3,4-dihydroxyphenylacetic acid (DOPAC), and NE were analysed in telencephalon, cerebellum, optic tectum and brainstem samples using high performance liquid chromatography with electrochemical detection (HPLC-EC) as described by Höglund et al. (2000). The concentrations were standardized against the weight of the brain tissues. The ratios of 5-HIAA to 5-HT concentration and DOPAC to DA concentration were calculated and used as an index of serotonergic and dopaminergic activity, respectively (Shannon et al. 1986).

\section{Behavioural observations}

In the analysis of the behaviour during treatment with anaesthetics we recorded: (1) activity until loss of equilibrium [anaesthesia stage III plane 1 (Zahl et al. 2012)] monitored by dividing the anaesthetization bucket into quadrants via a cross and counting number of crossed lines per second, (2) latency to loss of equilibrium, and (3) latency to anaesthesia [anaesthesia stage III plane 2 (Zahl et al. 2012)] defined as lost equilibrium and no response to three repeated tactile stimuli. This was done by one observer $(\mathrm{MH})$.

\section{Statistical analyses}

Normally distributed data were analysed using $t$-test, ANOVA or repeated measures ANOVA with LSD post hoc tests. Further, correlations between behaviour, physiology and pigmentation were investigated using either Pearson correlation test if data were normally distributed or Spearman rank correlation test if they were not. All

Table 1 ANOVA showing the effect of treatment (AA, AN, NA, $\mathrm{NN}$, A is for anaesthetic, $\mathrm{N}$ for non-anaesthetic, and procedure for counting spots was done on two consecutive days for all statistics were performed in IBM SPSS Statistics 20 (IBM Corporation, New York, USA) and data are presented as mean $\pm \mathrm{SEM}$ if not stated otherwise.

\section{Results}

\section{Carotenoid pigmentation}

The number of carotenoid spots on the right side differed between treatment groups on day 9 (Table 1, Fig. 2). The charr in group AA had more spots than the groups AN and NN, but not more spots than the NA group. However, on day 9 no difference was seen in spots on the left side (Table 1, Fig. 1). The number of carotenoid spots did not differ between treatment groups on either side on day 1 , or on day 8 (Table 1). When considering the photographing procedures separately, that is day 8 and 9 , there were some differences between anaesthetized and non-anaesthetized charr. Number of spots on the right side were higher in anaesthetized compared to non-anaesthetized charr on day $9(t$-test, $P=0.005)$, and a similar trend was seen on day 8 ( $t$-test, $P=0.059$ ) (see Fig. 3 ). On the left side, a similar trend could be noted on day 9 ( $t$-test, $P=0.069)$ but not on day 8 ( $t$-test, $P=0.262$ ) (see Fig. 2 ). All data are available in the Supplemental table 1.

Considering a change over time in the number of spots, both the AA group and the NN group differed in number of spots on the right side (Table 2). In the AA group there were more spots on the right side on day 8 compared to day 1 , whereas in the $\mathrm{NN}$ group there were less spots on the right side on day 9 compared to day 1 (Table 2). On the right side, no difference was evident in the AN group or in the NA group. Additionally, no difference could be seen on the left side in any group (Table 2).

individuals) on the number of spots on left and right side, calculated from photographs taken on day 1,8 , and 9

\begin{tabular}{|c|c|c|c|c|c|c|}
\hline & \multicolumn{3}{|c|}{ Right side } & \multicolumn{3}{|c|}{ Left side } \\
\hline & $\mathrm{F}_{3,28}$ & $\mathrm{P}$ & Post-hoc & $\mathrm{F}_{3,28}$ & $\mathrm{P}$ & Post-hoc \\
\hline Day 1 & 1.128 & 0.355 & - & 1.084 & 0.372 & - \\
\hline Day 8 & 2.771 & 0.060 & - & 1.759 & 0.178 & - \\
\hline Day 9 & 4.417 & 0.012 & $\mathrm{AA}>\mathrm{AN}, \mathrm{NN}$ & 2.060 & 0.128 & - \\
\hline
\end{tabular}

Post-hoc column show significant differences (LSD, P < 0.05) between treatments. Days were analysed separately

Significant results are shown in bold font 


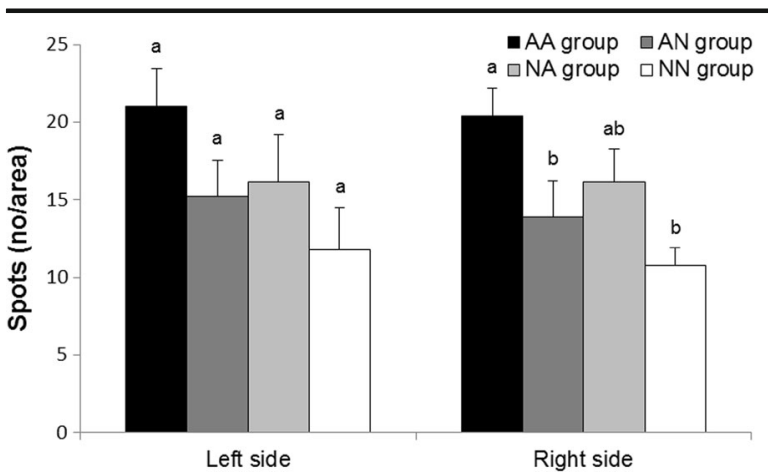

Fig. 2 Number of spots on day 9 on left and right side of each treatment ( $\mathrm{A}$ is for anaesthetic, $\mathrm{N}$ for non-anaesthetic, and procedure for counting spots was done on two consecutive days for all individuals). Different superscript capitals indicates significant differences (LSD post hoc, $\mathrm{P}<0.05$ ). Values are mean \pm S.E.M and $N=8$ for all treatments

There were several correlations between spots on separate days and sides (see Table 3). For instance, on the same day left and right side spots were positively correlated with spots on the other side on day 1, 8 and 9 . The spots on the right side were also positively correlated across all days (day 1, 8 and 9). Additionally, on day 8 and 9, left and right side spots were all positively correlated with each other. Finally, spots on the left side on day 1 were positively correlated with spots on the right side on day 9 .

\section{Physiological parameters}

Concerning monoamines, DOPAC concentration in telencephalon differed between treatments (ANOVA, $\mathrm{F}_{3,27}=3.941, P=0.019$; see Fig. 4), with the NA group having higher concentration of DOPAC than group AN (LSD post hoc, $P=0.004$ ) and NN (LSD post hoc,

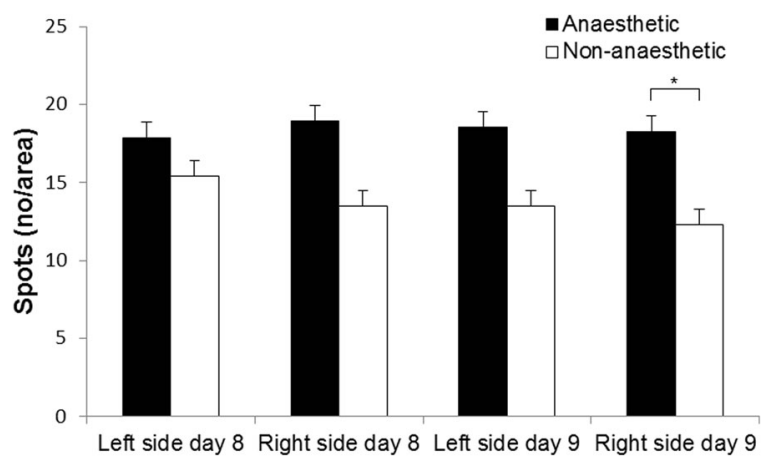

Fig. 3 Number of spots on day 8 and 9 on left and right side of anaesthetised $(\mathrm{A})$ and non-anaesthetised $(\mathrm{N})$ individuals. * indicates significant differences $(t$-test, $\mathrm{P}<0.05)$. Values are mean \pm S.E.M and $N=16$ for all treatments
Table 2 Repeated measures ANOVA on number of spots on left and right side, from photographs taken on day 1,8 , and 9

\begin{tabular}{|c|c|c|c|c|c|c|}
\hline & \multicolumn{3}{|c|}{ Right side } & \multicolumn{3}{|c|}{ Left side } \\
\hline & $\mathrm{F}_{2,14}$ & $\mathrm{P}$ & Post-hoc & $\mathrm{F}_{2,14}$ & $\mathrm{P}$ & Post-hoc \\
\hline AA & 4.341 & 0.034 & $8>1$ & 0.005 & 0.995 & - \\
\hline $\mathrm{AN}$ & 0.349 & 0.712 & - & 0.180 & 0.837 & - \\
\hline NA & 1.627 & 0.232 & - & 0.041 & 0.960 & - \\
\hline $\mathrm{NN}$ & 3.848 & 0.047 & $9<1$ & 1.363 & 0.288 & - \\
\hline
\end{tabular}

Post-hoc column show significant differences between days (LSD, $\mathrm{P}<0.05$ ). Treatments (AA, AN, NA, NN, A is for anaesthetic, $\mathrm{N}$ for non-anaesthetic, and procedure for counting spots was done on two consecutive days for all individuals) were analysed separately

$P=0.011$ ), but not AA (LSD post hoc, $P=0.064$ ). However, no other differences in monoamines between treatments were evident (see Table 4). Similarly, there were no differences in plasma cortisol between the groups (ANOVA, $\mathrm{F}_{3,28}=2.407, P=0.088$; see Fig. 5).

Several associations between monoamines and the carotenoid pigmentation on day 9 were noted in the treatment groups. For instance, in the AA group spots on the left side on day 9 were positively correlated to 5HIAA in the optic tectum (Pearson correlation, $\mathrm{r}=0.859, P=0.006, N=8)$. In the NA group spots on the right side on day 9 were positively correlated to DOPAC in the telencephalon (Pearson correlation, $\mathrm{r}=0.817, P=0.013, N=8$ ), and negatively correlated to 5-HT in the telencephalon (Pearson correlation, $\mathrm{r}=-0.739, P=0.036, N=8)$. In the NN group spots on the right side on day 9 were negatively correlated with DA in the brainstem (Pearson correlation, $r=-0.772$, $P=0.025, N=8)$. However, no associations were found in the AN group.

\section{Behaviour}

During the acclimation all fish started eating. There were no behavioural differences between the groups during anaesthetisation (see Table 5). However, behaviour during anaesthetisation was associated with carotenoid pigmentation. Spots on the left side on day 1 (Pearson correlation, $\mathrm{r}=0.539, P=0.031, N=16$ ) and on day 8 (Pearson correlation, $\mathrm{r}=0.591, P=0.016$, $N=16)$ were positively correlated to activity until loss of equilibrium on day 8 . Additionally, there were some associations within the treatment groups. For instance, in the NA group spots on the left side on day 1 were positively correlated with activity until loss of 
Table 3 The Pearson correlation coefficients and P-value for number of carotenoid spots of Arctic charr after handling stress

\begin{tabular}{|c|c|c|c|c|c|c|c|}
\hline & $\begin{array}{l}\text { Day } 1 \text {, } \\
\text { Left side }\end{array}$ & $\begin{array}{l}\text { Day 1, } \\
\text { Right side }\end{array}$ & $\begin{array}{l}\text { Day } 8, \\
\text { Left side }\end{array}$ & $\begin{array}{l}\text { Day } 8, \\
\text { Right side }\end{array}$ & $\begin{array}{l}\text { Day } 9 \text {, } \\
\text { Left side }\end{array}$ & $\begin{array}{l}\text { Day } 9, \\
\text { Right side }\end{array}$ & Mean \pm S.D. (N) \\
\hline Day 1, Left side & 1.00 & & & & & & $16.84 \pm 8.44$ \\
\hline Day 1, Right side & $0.478, P=0.006$ & 1.00 & & & & & $14.97 \pm 6.39(32)$ \\
\hline Day 8, Left side & $0.318, \mathrm{P}=0.077$ & $0.276, P=0.126$ & 1.00 & & & & $16.66 \pm 6.06(32)$ \\
\hline Day 8 , Right side & $0.248, \mathrm{P}=0.170$ & $0.507, P=0.003$ & $0.509, P=0.003$ & 1.00 & & & $16.22 \pm 8.19$ \\
\hline Day 9, Left side & $0.303, P=0.092$ & $0.347, \mathrm{P}=0.052$ & $0.574, P=0.001$ & $0.588, \mathrm{P}<0.001$ & 1.00 & & $16.03 \pm 7.89(32)$ \\
\hline Day 9, Right side & $0.377, P=0.034$ & $0.416, P=0.018$ & $0.541, P=0.001$ & $0.689, \mathrm{P}<0.001$ & $0.743, \mathrm{P}<0.001$ & 1.00 & $15.28 \pm 6.29$ \\
\hline
\end{tabular}

Significant results are shown in bold font

equilibrium (Pearson correlation, $\mathrm{r}=0.837, P=0.009$, $N=8$ ), and with time until anaesthesia (Pearson correlation, $\mathrm{r}=0.757, P=0.030, N=8$ ) on day 9 . In the AA group, spots on the left side on day 8 were positively correlated with activity until loss of equilibrium on day 8 (Pearson correlation, $\mathrm{r}=0.857, P=0.007, N=8$ ), and time until loss of equilibrium (Pearson correlation, $\mathrm{r}=$ $0.751, P=0.032, N=8$ ) on day 9 .

\section{Discussion}

The main purpose of this study was to investigate the possibility to use carotenoid pigmentation in Arctic charr as a tool for evaluating stress responsiveness, especially under normal maintenance, i.e. with the use of the anaesthetic MS-222. Some of the results indicate that anaesthetization affects number of spots. For instance, the group that was anaesthetized twice (AA) had more spots on the right side than the groups not

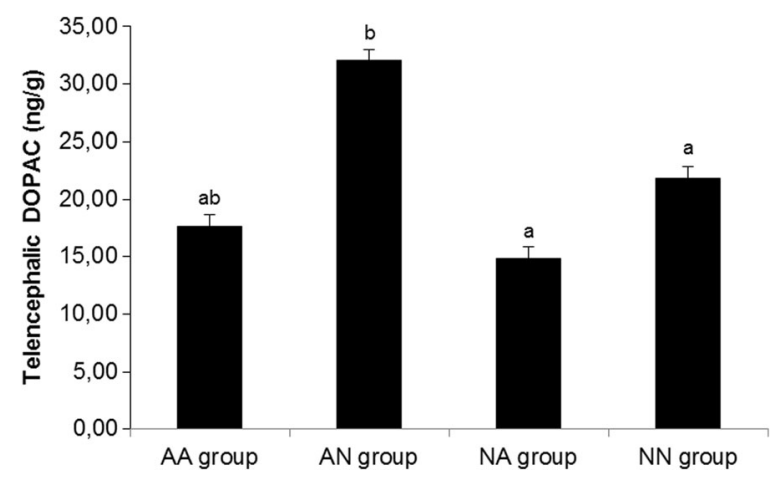

Fig. 4 DOPAC levels in telencephalon after treatments (A is for anaesthetic and $\mathrm{N}$ for non-anaesthetic). Different superscript capitals indicates significant differences (LSD post hoc, $\mathrm{P}<0.05$ ). Values are mean \pm S.E.M and $N=8$ for all treatments except for group NA $(N=7)$ anaesthetized the last day (AN and NN). This was also evident when considering spots on the right side in anaesthetized compared to not anaesthetized on day 9, when anaesthetized charr had more spots, and a similar trend was found on day 8 . Further this trend was to some extent evident in spots on the left side on day 9 but not on day 8. This is in line with the report that anaesthesia induced carotenoid spots in Arctic charr (Backström et al. 2015b), as well as the reported stress-inducing effect of the anaesthetic MS-222 in teleost fish (Zahl et al. 2010; Readman et al. 2013). Thus the anaesthetic treatment before photographing could arguably be the more stressful handling. This is further corroborated by the higher dopaminergic activity in telencephalon of anaesthetised individuals the last day in our study, which could be indicative for higher stress responsiveness (Fuchs and Flugge 2003; Höglund et al. 2005; Backström et al. 2011). Thus there are indications of both spots and physiological stress being induced by anaesthetics. However, we could not see any differences in the plasma cortisol levels which was expected from an earlier study (Backström et al. 2015b). Plasma for measuring cortisol was taken quickly (range of time until anaesthesia 60-185 s and an approximate additional $30 \mathrm{~s}$ for photographing) after netting fish from isolation aquaria, and is therefore not expected to show a full stress response (Wendelaar Bonga 1997; Cockrem 2013; Gesto et al. 2013; López-Patiño et al. 2014). Since both treatments are stressful small differences were expected. Further, in this study there were three similar stressful events consecutively and thus stress could be predictable. Predictable stress has been shown to reduce the cortisol response (Galhardo et al. 2011), and a comparison to an earlier study (Backström et al. 2015b) indicates that there is a lower elevation of cortisol in our study. However, the number of repeated stimuli is low so it might not be a plausible explanation. 
Table 4 Concentrations of NE, DOPAC, DA, 5-HIAA and 5-HT, and DOPAC/DA and 5-HIAA/5-HT ratios in the brainstem, cerebellum, optic tectum, and telencephalon in all of the Arctic charr combined after handling stress

\begin{tabular}{llllllll}
\hline Brain part & NE (ng/g) & DOPAC (ng/g) & DA (ng/g) & DOPAC/DA*10 & 5-HIAA (ng/g) & 5-HT (ng/g) & 5-HIAA/5-HT*10-3 \\
\hline Brain stem & $63 \pm 11(32)$ & $14 \pm 9(32)$ & $59 \pm 37(32)$ & $264 \pm 113(32)$ & $4 \pm 1(31)$ & $36 \pm 9(32)$ & $100 \pm 30(31)$ \\
Cerebellum & $18 \pm 5(32)$ & $32 \pm 25(32)$ & n.d. & n.d. & n.d. & n.d. & n.d. \\
Optic tectum & $50 \pm 11(32)$ & $25 \pm 18(32)$ & $12 \pm 18(32)$ & $3129 \pm 1432(32)$ & $3 \pm 1(32)$ & $20 \pm 7(32)$ & $163 \pm 42(32)$ \\
Telencephalon & $96 \pm 32(32)$ & $22 \pm 12(31)$ & $48 \pm 37(32)$ & $673 \pm 546(32)$ & $6 \pm 2(32)$ & $39 \pm 15(32)$ & $168 \pm 71(31)$ \\
\hline
\end{tabular}

Values are mean \pm S.D. (N). n.d. indicates that monoamines were not detected or too few to be analysed further

Some of the monoaminergic activities were associated with carotenoid pigmentation. Serotonergic activity in the optic tectum was positively correlated with left side spots in the AA group, supposedly the most stressed group (see above). During stress the serotonergic activity increases (Winberg and Nilsson 1993; Summers and Winberg 2006), and thus this follows predictions and supports earlier results on telencephalic serotonergic activity association with left side spots in social dominants (Backström et al. 2015c). Dopaminergic activity in telencephalon was positively correlated to right side spots in the PA group. This could be indicative for higher stress responsiveness (Fuchs and Flugge 2003; Höglund et al. 2005; Backström et al. 2011), and is similar to an earlier study of social subordinate Arctic charr concerning dopaminergic activity in optic tectum and its association with right side spots (Backström et al. 2015c), although there is a temporal difference in sampling time. The same group also had a positive correlation between left side spots and activity during anaesthesia. Thus, the increased activity was reflected in both DA concentrations and spots.

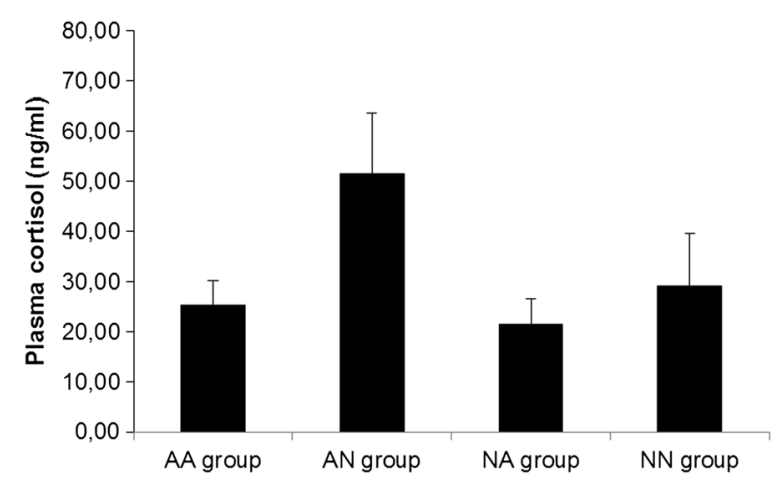

Fig. 5 Plasma cortisol levels after treatments (A is for anaesthetic and $\mathrm{N}$ for non-anaesthetic). Values are mean \pm S.E.M and $N=8$ for all treatments
However, all individuals were also tested the day before and therefore might have a lingering stress effects.

Behaviour during stress was associated to carotenoid pigmentation in this study. During the anaesthetization, activity until loss of equilibrium was higher in individuals with more spots on the left side. Similarly, in some groups (NA and AA) individuals with more left side spots took longer time until loss of equilibrium. This is the opposite result compared to an earlier report that showed a negative correlation between spots and both activity and time until loss of equilibrium during anaesthetization comparing the effect of several anaesthetics (Backström et al. 2015b). This was however not observed for MS-222 (Backström et al. 2015b), that was used in this study. This difference could be the result of predictable stress (see above). Further, the protocols were slightly different with two more photographing sessions (in total three) in this study, which could influence the behaviour.

Thus, several of the results from this study indicate that MS-222 affect the spots and therefore would not be recommended to be used when evaluating stress responsiveness. However, carotenoid spots were highly correlated between days and especially on the last days, during the treatments. Further, there also seems to be a lateralized effect of the carotenoid pigmentation. It has been suggested that the left side is associated to stress responsiveness whereas the right side is associated to aggressiveness (Backström et al. 2015a; Backström et al. 2015c). The left side association to stress responsiveness seems to be corroborated in this study. For instance, activity during anaesthesia, which indicates more stress (see above), was positively correlated to left side spots. Additionally, serotonergic activity in the optic tectum of the group AA was positively associated with the left side spots. Since increased serotonergic activity is associated with stress response (see above), this follows predictions. However, 5-HT in the optic 
Table 5 The behaviour during anaesthesia in different treatment groups (A is for anaesthetic, $\mathrm{N}$ for non-anaesthetic, and procedure for counting spots was done on two consecutive days for all individuals) of juvenile Arctic charr after handling stress

\begin{tabular}{|c|c|c|c|c|}
\hline Treatment & AA group & AN group & NA group & NN group \\
\hline \multicolumn{5}{|l|}{ Day 8} \\
\hline Activity until loss of equilibrium, stage III plane 1 (no/s) & $0.17 \pm 0.10(8)$ & $0.16 \pm 0.06(8)$ & - & - \\
\hline Time until loss of equilibrium, stage III plane 1 (s) & $42.0 \pm 9.9(8)$ & $49.9 \pm 9.7(8)$ & - & - \\
\hline Time until anaesthesia, stage III plane 2 (s) & $113.3 \pm 57.7(8)$ & $107.4 \pm 32.8(8)$ & - & - \\
\hline \multicolumn{5}{|l|}{ Day 9} \\
\hline Activity until loss of equilibrium, stage III plane 1 (no/s) & $0.15 \pm 0.11(8)$ & - & $0.09 \pm 0.05(8)$ & - \\
\hline Time until loss of equilibrium, stage III plane 1 (s) & $39.1 \pm 10.7(8)$ & - & $47.8 \pm 8.2(8)$ & - \\
\hline Time until anaesthesia, stage III plane 2 (s) & $79.1 \pm 10.7(8)$ & - & $106.5 \pm 34.6(8)$ & - \\
\hline
\end{tabular}

Loss of equilibrium, stage III plane 1, and anaesthesia, stage III plane 2 are based upon definitions from Zahl et al. (2012), see text for further information. Values are mean \pm S.D. $(\mathrm{N})$

tectum was negatively correlated to the right side spots of group NA. In the groups NA and NN, dopaminergic activity in the optic tectum was positively correlated to right side spots. Thus there are some connections between right side spots and the monoaminergic systems as well.

The mechanism controlling size and number of carotenoid spots is not known at present. However, since it has been shown earlier that carotenoid spot patterns can change rapidly (Backström et al. 2015b), it is probably an effect of physiological colour change (Mills and Patterson 2009; Nilsson Sköld et al. 2013). One of the main factors controlling physiological colour change is $\alpha$-melanocyte-stimulating hormone ( $\alpha$-MSH) (Mills and Patterson 2009; Nilsson Sköld et al. 2013), and it has been shown to be involved in skin darkening of Arctic charr during social interactions (Höglund et al. 2000; Höglund et al. 2002). Thus it seems likely that this process can also be involved in and have an effect on the carotenoid spots. The contrast between the spots and the background coloration of the skin could be an effect of this skin darkening, but in an earlier study we found it unlikely (Backström et al. 2015a). Further, since there also seems to be a link between carotenoid spots counted the day before and the stress responsiveness the following day (Backström et al. 2014), the physiological colour change is not the only factor involved in the association between carotenoid spots and stress responsiveness. Therefore the mechanism of the change as well as the origin of the spots needs further studies.

In conclusion, this study adds further support to the hypothesis that carotenoid spots, at least on the left side, can predict stress responsiveness in Arctic charr. This is evident in behavioural responses as well as monoaminergic responses. Finally, it seems plausible that using anaesthetics for photographing is possible without confounding the results, however careful planning is needed and especial attention concerning which side to use.

Acknowledgments The methodology of this study was approved by the Swedish University of Agricultural Sciences Ethical Committee. Financing was granted by the Swedish Research Council Formas (Dnr 2011-888).

Authors' contributions TB and MH designed and performed the study. TB carried out cortisol and monoamine analysis, and drafted the manuscript. EB, JN and CM participated in design of the study. SW helped with monoamine analysis. All authors participated in revising the manuscript and gave final approval for publication.

\section{Compliance with ethical standards}

Competing interests We have no competing interests.

Open Access This article is distributed under the terms of the Creative Commons Attribution 4.0 International License (http:// creativecommons.org/licenses/by/4.0/), which permits unrestricted use, distribution, and reproduction in any medium, provided you give appropriate credit to the original author(s) and the source, provide a link to the Creative Commons license, and indicate if changes were made.

\section{References}

Backström T, Brännäs E, Nilsson J, Magnhagen C (2014) Behaviour, physiology and carotenoid pigmentation in Arctic charr Salvelinus alpinus. J Fish Biol 84(1):1-9. doi:10.1111/jfb.12240 
Backström T, Heynen M, Brännäs E, Nilsson J, Magnhagen C (2015a) Dominance and stress signalling of carotenoid pigmentation in Arctic charr (Salvelinus alpinus): lateralization effects? Physiol Behav 138:52-57. doi:10.1016/j. physbeh.2014.10.003

Backström T, Heynen M, Brännäs E, Nilsson J, Magnhagen C (2015b) The effect of anesthetics on carotenoid pigmentation and behavior in Arctic char (Salvelinus alpinus). J Vet Behav: Clin Appl Res 10(2):179-184. doi:10.1016/j. jveb.2014.11.007

Backström T, Heynen M, Brännäs E, Nilsson J, Winberg S, Magnhagen C (2015c) Social stress effects on pigmentation and monoamines in Arctic charr. Behav Brain Res 291:103107. doi:10.1016/j.bbr.2015.05.011

Backström T, Schjolden J, Øverli Ø, Thörnqvist PO, Winberg S (2011) Stress effects on AVT and CRF systems in two strains of rainbow trout (Oncorhynchus mykiss) divergent in stress responsiveness. Horm Behav 59(1):180-186. doi:10.1016/j. yhbeh.2010.11.008

Blanchard RJ, McKittrick CR, Blanchard DC (2001) Animal models of social stress: effects on behavior and brain neurochemical systems. Physiol Behav 73(3):261-271. doi:10.1016/S0031-9384(01)00449-8

Castanheira MF, et al. (2015) Coping styles in farmed fish: consequences for aquaculture. Rev Aquacult:n/a-n/a doi:10.1111 raq. 12100

Cockrem JF (2013) Individual variation in glucocorticoid stress responses in animals. Gen Comp Endocrinol 181:45-58. doi:10.1016/j.ygcen.2012.11.025

Conrad JL, Weinersmith KL, Brodin T, Saltz JB, Sih A (2011) Behavioural syndromes in fishes: a review with implications for ecology and fisheries management. J Fish Biol 78(2): 395-435

Duarte CM, Marbá N, Holmer M (2007) Rapid domestication of marine species. Science 316(5823):382-383. doi:10.1126 /science. 1138042

Ducrest A-L, Keller L, Roulin A (2008) Pleiotropy in the melanocortin system, coloration and behavioural syndromes. Trends Ecol Evol 23(9):502-510. doi:10.1016/j. tree.2008.06.001

Eaton L, Sloman KA (2011) Subordinate brown trout exaggerate social signalling in turbid conditions. Anim Behav 81(3): 603-608. doi:10.1016/j.anbehav.2010.12.005

Erhard HW, Mendl M, Ashley DD (1997) Individual aggressiveness of pigs can be measured and used to reduce aggression after mixing. Appl Anim Behav Sci 54(2-3):137-151. doi:10.1016/S0168-1591(97)00068-3

Fuchs E, Flugge G (2003) Chronic social stress: effects on limbic brain structures. physiology \& behavior. a tribute to Paul MacLean. Neurobiol Relev Soc Behav 79(3):417-427. doi:10.1016/S0031-9384(03)00161-6

Galhardo L, Vital J, Oliveira RF (2011) The role of predictability in the stress response of a cichlid fish. Physiol Behav 102(34):367-372. doi:10.1016/j.physbeh.2010.11.035

Gesto M, López-Patiño MA, Hernández J, Soengas JL, Míguez JM (2013) The response of brain serotonergic and dopaminergic systems to an acute stressor in rainbow trout: a time course study. J Exp Biol 216(23):4435-4442. doi:10.1242/jeb.091751

Hill GE (2011) Condition-dependent traits as signals of the functionality of vital cellular processes. Ecol Lett 14(7):625-634. doi:10.1111/j.1461-0248.2011.01622.x
Huntingford F, Adams C (2005) Behavioural syndromes in farmed fish: implications for production and welfare. Behaviour 142(9-10):1207-1221. doi:10.1163/156853905774539382

Huntingford FA et al (2006) Current issues in fish welfare. J Fish Biol 68(2):332-372. doi:10.1111/j.00221112.2006.001046.x

Höglund E, Balm P, Winberg S (2000) Skin darkening, a potential social signal in subordinate arctic charr (Salvelinus alpinus): the regulatory role of brain monoamines and proopiomelanocortin-derived peptides. J Exp Biol 203(11): 1711-1721

Höglund E, Balm PHM, Winberg S (2002) Behavioural and neuroendocrine effects of environmental background colour and social interaction in Arctic charr (Salvelinus alpinus). J Exp Biol 205(16):2535-2543

Höglund E, Weltzien F-A, Schjolden J, Winberg S, Ursin H, Døving KB (2005) Avoidance behavior and brain monoamines in fish. Brain Res 1032(1-2):104-110. doi:10.1016 /j.brainres.2004.10.050

Kittilsen S et al (2009) Melanin-based skin spots reflect stress responsiveness in salmonid fish. Horm Behav 56(3):292298. doi:10.1016/j.yhbeh.2009.06.006

Koolhaas JM, de Boer SF, Buwalda B, van Reenen K (2007) Individual variation in coping with stress: a multidimensional approach of ultimate and proximate mechanisms. Brain Behav Evol 70(4):218-226

Koolhaas JM, de Boer SF, Coppens CM, Buwalda B (2010) Neuroendocrinology of coping styles: Towards understanding the biology of individual variation. Front Neuroendocrinol 31(3):307-321. doi:10.1016/j. yfrne.2010.04.001

Koolhaas JM et al (1999) Coping styles in animals: current status in behavior and stress-physiology. Neurosci Biobehav Rev 23(7):925-935

López-Patiño MA, Hernández-Pérez J, Gesto M, Librán-Pérez M, Míguez JM, Soengas JL (2014) Short-term time course of liver metabolic response to acute handling stress in rainbow trout, Oncorhynchus mykiss. Comp Biochem Physiol A Mol Integr Physiol 168:40-49. doi:10.1016/j.cbpa.2013.10.027

Mills MG, Patterson LB (2009) Not just black and white: pigment pattern development and evolution in vertebrates. Semin Cell Dev Biol 20(1):72-81. doi:10.1016/j.semcdb.2008.11.012

Nilsson J, Brännäs E, Eriksson LO (2010) The Swedish Arctic charr breeding programme. Hydrobiologia 650(1):275-282. doi:10.1007/s10750-010-0232-9

Nilsson Sköld H, Aspengren S, Wallin M (2013) Rapid color change in fish and amphibians - function, regulation, and emerging applications. Pigment Cell Melanoma Res 26(1): 29-38. doi:10.1111/pcmr.12040

Øverli Ø, Winberg S, Pottinger TG (2005) Behavioral and neuroendocrine correlates of selection for stress responsiveness in rainbow trout - a review. Integr Comp Biol 45(3):463-474

O'Connor KI, Metcalfe NB, Taylor AC (1999) Does darkening signal submission in territorial contests between juvenile Atlantic salmon, Salmo salar ? Anim Behav 58(6):12691276. doi:10.1006/anbe.1999.1260

O'Connor KI, Metcalfe NB, Taylor AC (2000) Familiarity influences body darkening in territorial disputes between juvenile salmon. Anim Behav 59(6):1095-1101. doi:10.1006 /anbe.2000.1401 
Readman GD, Owen SF, Murrell JC, Knowles TG (2013) Do fish perceive anaesthetics as aversive? PLoS One 8(9):e73773. doi:10.1371/journal.pone. 0073773

Schjolden J, Winberg S (2007) Genetically determined variation in stress responsiveness in rainbow trout: behavior and neurobiology. Brain Behav Evol 70(4):227-238

Shannon NJ, Gunnet JW, Moore KE (1986) A comparison of biochemical indices of 5-hydroxytryptaminergic neuronal activity following electrical stimulation of the dorsal Raphe nucleus. J Neurochem 47(3):958-965. doi:10.1111/j.14714159.1986.tb00704.x

Summers CH, Winberg S (2006) Interactions between the neural regulation of stress and aggression. J Exp Biol 209(23): $4581-4589$
Wendelaar Bonga SE (1997) The stress response in fish. Physiol Rev 77(3):591-625

Winberg S, Nilsson GE (1993) Roles of brain monoamine neurotransmitters in agonistic behaviour and stress reactions, with particular reference to fish. ComparaT Biochem Physiol Part C: Comparat Pharmacol Toxicol 106(3):597-614

Zahl I, Kiessling A, Samuelsen O, Olsen R (2010) Anesthesia induces stress in Atlantic salmon (Salmo salar), Atlantic cod (Gadus morhua) and Atlantic halibut (Hippoglossus hippoglossus). Fish Physiol Biochem 36(3):719-730. doi:10.1007/s10695-009-9346-2

Zahl I, Samuelsen O, Kiessling A (2012) Anaesthesia of farmed fish: implications for welfare. Fish Physiol Biochem 38(1): 201-218. doi:10.1007/s10695-011-9565-1 